\title{
Receptor abuse-dependent antagonism for neuroprotection
}

Hari Manev

A recent Perspective article ${ }^{1}$ on strategies for the development of clinically well-tolerated novel neuroprotective drugs focuses on the principle that drugs should interact with their target only during states of pathological activation, and not interfere with normal functions. The term 'pathologically activated therapeutics' (PAT) has been coined to describe such drugs.

It should be acknowledged that a similar principle for developing neuroprotective drugs was introduced almost 20 years ago under a different acronym, RADA ${ }^{2}$. RADA stands for 'receptor abuse-dependent antagonism' and describes a set of guidelines for designing neuroprotective drugs capable of selectively blocking the pathological effects of the neurotransmitter glutamate in neurons surrounding ischemic/injured brain areas while leaving the physiological actions of glutamate in nonlesioned areas of the brain unaffected. This concept is based on the notion that during ischemia and in brain injury glutamate concentrations at synapses surrounding a lesion are increased for prolonged periods of time and might lead to the abusive stimulation of glutamate receptors. It was proposed that an essential component of RADA is to delineate receptor-induced intracellular events that are specific to receptor abuse. This is also the cornerstone of PAT. As brain functioning, with few exceptions, almost exclusively relies on receptor-mediated signalling, it is not surprising that the neuroprotective PAT described in the Perspective article include RADA.

The more recent PAT and the previous RADA strategies remain valid in neuroprotective drug research. Nevertheless, their clinical success will be decided by a successful identification of pathology-specific cellular signalling molecules. Perhaps the brain ischemia/injury-specific metabolomics studies will provide the necessary impetus.

Hari Manev is at The Psychiatric Institute, Department of Psychiatry, University of Illinois at Chicago, Chicago, Illinois 60612, USA.

1. Lipton, S. A. Pathologically activated therapeutics for neuroprotection. Nature Rev. Neurosci. 8, 803-808 (2007)

2. Manev, H., Costa, E., Wroblewski, J. T. \& Guidotti, A Abusive stimulation of excitatory amino acid receptors: a strategy to limit neurotoxicity. FASEB J. 4 2789-2797 (1990). 